\title{
Metabolomic profile of colorectal cancer patients and its clinical implications
}

\author{
CLAUDIU RĂCHIERIU ${ }^{1,2}$, FLORIN GRAUR ${ }^{2,3 *}$, EMIL MOIS ${ }^{2,3}$, CARMEN \\ SOCACIU $^{4}$, DAN TUDOR ENIU ${ }^{2,5}$, NADIM AL HAJJAR ${ }^{2,3}$
}

${ }^{1}$ Surgery Department. County Hospital Alba, Bld. Revolutiei 1989, No. 23, Alba Iulia, Romania ${ }^{2}$ Faculty of Medicine, Iuliu Hatieganu University of Medicine and Pharmacy, Cluj-Napoca, Romania ${ }^{3}$ Regional Institute of Gastroenterology and Hepatology "Octavian Fodor", Cluj-Napoca, Romania

${ }^{4}$ Research Center for Applied Biotechnology in Diagnosis and Molecular Therapy, BIODIATECH Cluj-Napoca, Romania

${ }^{5}$ Department of Surgical and Gynecological Oncology, Ion Chiricuta Oncologic Institute, 400015 Cluj-Napoca, Romania

\begin{abstract}
Background: Colorectal Cancer (CRC) is a great public health issue and the outcomes of treatment depends on early diagnosis. Metabolomics may provide biomarkers for early diagnosis, staging, prognosis and follow-up.

Methods: The authors searched for the results of the published studies existing in medical databases (PubMed) for all the changes in the main metabolic pathways (carbohydrate, lipid, aminoacid, nucleotide and other important metabolites) of the CRC patients and how the metabolic changes can be used as biomarkers, for tumor staging, prognosis and follow-up.

Results: While most of the metabolites values in biological fluids or tissue samples are modified (either increased or decreased) and the results are usually constant across the studies it seems that only patterns of metabolites (fingerprints of 5, 15 or even 30 metabolites) can be used for the regarding issues mentioned above.

Conclusion: Some studies conclude that some metabolomic models are statistically much better than current existing markers and may become screening methods in the near future.
\end{abstract}

Keywords Metabolomics, colorectal cancer, biomarkers.

To cite this article: RĂCHIERIU C, GRAUR F, MOIS E, SOCACIU C, ENIU DT, AL HAJJAR N. Metabolomic profile of colorectal cancer patients and its clinical implications. Rom Biotechnol Lett. 2020; 25(6): 2045-2054. DOI: 10.25083/rbl/25.6/2045.2054

GRAUR F, RĂCHIERIU C, Faculty of Medicine, Iuliu Hatieganu University of Medicine and Pharmacy, Str. Victor Babeş Nr. 8, 400012, Cluj-Napoca, Romania Tel.:+40744807242 (G.F.), +40746151201 (R.C.)

E-mail: i graurf@yahoo.com crachieriu@yahoo.com 


\section{Introduction}

Colorectal Cancer (CRC) is a great public health issue, particularly in Western Countries but also in developing countries, as the population borrows the western food habits. The American Cancer Society (ACS) reports in 2014 about 71800 men and 65000 women diagnosed with colorectal cancer in the United States of America. There were a quite similar number of males and females with colon cancer (around 48000), while the rectal cancer was more frequent in males compared with women in a 3:2 ratio (about 23380 vs 16620 cases). The number of deaths was about 26270 for males and 24040 for females. After a relatively ascending trendline of the incidence of CRC from 1975 towards the mid '80's, now there is a slow decreasing incidence, especially in the last decade, probably due to early detection and endoscopic resection of adenomatous polyps (premalignant conditions) and colorectal cancer screening [1]. ACS estimates in 2017 that approximately $4.6 \%$ of men ( 1 in 22 ) and $4.2 \%$ of women ( 1 in 24) will be diagnosed with CRC in their lifetime, around 95000 new cases of colon cancer and almost 40000 cases of new rectal cancer cases [2].

Despite all the progress of medical oncology related to the development of new cytostatics, new multidisciplinary protocols that include chemotherapy, radiotherapy and surgery, combined in different sequences and combinations depending on tumor stage, developing new molecules for biologic treatment (monoclonal antibodies), the main curative treatment remains surgery. This is addressed to the so called "surgical stages" (stage I-III) but is also potentially curative in advanced stages. Although most metastatic patients will receive only palliative therapy, there are cases that can benefit from aggressive surgery with curative intend. The prototype situation is obtaining long disease-free interval or even healing of some cases with limited metastatic disease of the liver. Surgical intervention on patients with limited intrahepatic metastatic disease and without extrahepatic metastasis can lead to a mean survival interval of 40 to 58 months and a 5-year survival rate of 40-58\% [3]. So it's important to distinguish between this cases. Unfortunately only $30 \%$ of the patients with liver metastasis are operable [3] and of these $30 \%$ many of them have occult metastasis, which will be obvious after some time. Tumor staging is performed by the radiologic methods available at this moment (ultrasound, CT-scan, magnetic resonance imaging - MRI, sometimes PET-CT) and later intra-operatively, sometimes using laparoscopy, and in the end by pathologic findings. One of the problems that concern the medical world is finding biomarkers in order to distinguish between the cases that can be potentially cured by surgical treatment and those with occult metastasis. One of the possible solutions offered by the last decade literature might be "metabolomics".

Terminology. There is a small controversy in the literature regarding the terminology. There are two terms: "metabolomics" and "metabonomics" with specific definitions but in the literature the two of them are seldom confused, overlaid or improperly used. According to medical dictionaries the first term means: "the study of the small molecules, such as sugars and amino acids, that are substrates, products, or intermediates of metabolic reactions in an organism" [4]. Another definition is: "Metabolomics is the systematic study of the unique chemical fingerprints of small-molecules, or metabolite profiles, that are related to a variety of cellular metabolic processes in a cell, organ, or organism" [5]. On the other hand "metabonomics" is considered "the metabolic response of living systems to pathophysiologic stresses or genetic modification as measured in a quantitative and time-related fashion; sometimes used to draft dietary guidelines" [6]. According to the same source the "metabolome" is "the set of all metabolites in a particular organism, i.e., a statistical approximation of the organism's total metabolic potential under any condition" [7]. As a final statement the term "metabolomics" is rather related to a qualitative assessment of the entire metabolic profile (fingerprint), globally, while "metabonomics" represents a quantitative measurement of a response to a specific stimulus.

Metabolomic techniques. The field of molecular biology has had a fast progress in the last decade, with great implications in diagnosis, prognosis and follow-up of the surgical patients, particularly the oncologic ones. One of the new-born branches of molecular biology with a great potential on patient "staging" is metabolomics, which had an explosive development in the last decade [8]. It uses either MRI or chromatography coupled with mass spectrophotometry detection, in order to reveal the presence of the metabolites in the tissue samples obtained from surgical specimens or from biologic fluids (serum, urine). In some situations we can identify constellations, patterns of metabolites which can indicate the metabolic processes that are unfolding in the studied tissues [9]. Because of its high sensitivity and fast acquisition of data, mass spectrometry (MS), in combination with chromatography, plays an increasing important role in the field of metabolomics. There was a great progress regarding the technology ("the tools"), experimental models, software and databases. From qualitative detection methods and simple recognition of some metabolomic models we have evolved to quantitative "targeted" methods that are easy to reproduce and with trustworthy results. The new techniques of ambient ionization with quick evaporation or desorbent ionization allows "real time" direct MS, as well as new imaging techniques of MS [8].

\section{Methods}

The authors searched the PubMed scientific papers published before October 2018 related to the subject by entering the terms "colorectal cancer metabolomics", and applying the filter "humans". That search revealed 213 articles. The articles regarding the effects of various experimental treatments on Colorectal cancer cell lines were excluded, as were those that referred only marginally the metabolomics of CRC and were mainly related to other "omics" or genetics. The authors also excluded the papers that referred to just one specific metabolite modified in 
CRC or the specific metabolic changes caused by just one specific mutation. The search also included some experimental studies on CRC induced in rats and those articles were not included in this overview. Other studies related to obesity and diet and their possible implications in carcinogenesis in several cancers, not specifically CRC and were not considered important for the subject. Articles that referred to other cancers and not CRC (breast cancer, prostate cancer, esophageal and thyroid cancer), the metabolic changes in cancer cachexia, one article related to an international consensus meeting or articles related to specific devices used for studying metabolomics were also excluded. In the end 25 articles were searched by the authors regarding clinical applications, the different methabolic pathways affected by $\mathrm{CRC}$, predisposing factors and early diagnosis. The list of articles is found in Table 1.

Table 1. Articles included in our review

\begin{tabular}{|c|c|c|c|}
\hline No. & Author(s) & Title & Date \\
\hline 1. & Bathe OF, Farshidfar F & $\begin{array}{l}\text { From Genotype to Functional Phenotype: Unraveling the Metabolomic } \\
\text { Features of Colorectal Cancer. }\end{array}$ & 2014 \\
\hline 2. & $\begin{array}{l}\text { Farshidfar F, Weljie AM, Kopciuk K, Buie } \\
\text { WD, Maclean A, Dixon E, et al }\end{array}$ & $\begin{array}{l}\text { Serum metabolomic profile as a means to distinguish stage of colorectal } \\
\text { cancer. }\end{array}$ & 2012 \\
\hline 3. & $\begin{array}{l}\text { Tan B, Qiu Y, Zou X, Chen T, Xie G, Cheng } \\
\text { Y, et al. }\end{array}$ & $\begin{array}{l}\text { Metabonomics Identifies Serum Metabolite Markers of Colorectal } \\
\text { Cancer. }\end{array}$ & 2013 \\
\hline 4. & $\begin{array}{l}\text { Qiu Y, Cai G, Zhou B, Li D, Zhao A, Xie G, et } \\
\text { al. }\end{array}$ & $\begin{array}{l}\text { A Distinct Metabolic Signature of Human Colorectal Cancer with } \\
\text { Prognostic Potential. }\end{array}$ & 2014 \\
\hline 5. & \begin{tabular}{|l|} 
Muc-wierzgoń M, Nowakowska-zajdel E, \\
Dzięgielewska-gęsiak S, Kokot T. \\
\end{tabular} & $\begin{array}{l}\begin{array}{l}\text { Specific metabolic biomarkers as risk and prognostic factors in colorectal } \\
\text { cancer. }\end{array} \\
\end{array}$ & 2014 \\
\hline 6. & Beggs AD, Dilworth MP. & Surgery in the era of the 'omics revolution. & 2015 \\
\hline 7. & $\begin{array}{l}\text { Farshidfar F, Weljie AM, Kopciuk KA, } \\
\text { Hilsden R, Mcgregor SE, Buie WD, et al }\end{array}$ & $\begin{array}{l}\text { A validated metabolomic signature for colorectal cancer : exploration of } \\
\text { the clinical value of metabolomics. }\end{array}$ & 2016 \\
\hline 8. & $\begin{array}{l}\text { Zhang F, Zhang Y, Zhao W, Deng K, Wang Z. } \\
2017\end{array}$ & $\begin{array}{l}\text { Metabolomics for biomarker discovery in the diagnosis , prognosis, } \\
\text { survival and recurrence of colorectal cancer : a systematic review. }\end{array}$ & 2017 \\
\hline 9. & $\begin{array}{l}\text { Deng L, Fang H, Tso VK, Sun Y, Foshaug RR, } \\
\text { Krahn SC, et al. }\end{array}$ & $\begin{array}{l}\text { Clinical validation of a novel urine-based metabolomic test for the } \\
\text { detection of colonic polyps on Chinese population. }\end{array}$ & 2017 \\
\hline 10. & $\begin{array}{l}\text { Nishiumi S, Kobayashi T, Kawana S, Unno Y, } \\
\text { Sakai T, Okamoto K, et al. }\end{array}$ & $\begin{array}{l}\text { Investigations in the possibility of early detection of colorectal cancer by } \\
\text { gas chromatography/triple-quadrupole mass spectrometry }\end{array}$ & 2017 \\
\hline 11. & $\begin{array}{l}\text { Audet-delage Y, Rouleau M, Rouleau M, } \\
\text { Roberge J. }\end{array}$ & $\begin{array}{l}\text { Cross-Talk between Alternatively Spliced UGT1A Isoforms and Colon } \\
\text { Cancer Cell Metabolism. }\end{array}$ & 2017 \\
\hline 12. & $\begin{array}{l}\text { Jing Y, Wu X, Gao P, Fang Z, Wu J, Wang Q, } \\
\text { et al. }\end{array}$ & $\begin{array}{l}\text { Rapid differentiating colorectal cancer and colorectal polyp using dried } \\
\text { blood spot mass spectrometry metabolomic approach. }\end{array}$ & 2017 \\
\hline 13. & $\begin{array}{l}\text { Liesenfeld DB, Grapov D, Fahrmann JF, Salou } \\
\text { M, Scherer D, Toth R, et al. }\end{array}$ & $\begin{array}{l}\text { Metabolomics and transcriptomics identify pathway differences between } \\
\text { visceral and subcutaneous adipose tissue in colorectal cancer patients: } \\
\text { the ColoCare study. }\end{array}$ & 2015 \\
\hline 14. & $\begin{array}{l}\text { Kühn T, Floegel A, Sookthai D, Johnson T, } \\
\text { Rolle-Kampczyk U, Otto W, et al. } \\
\end{array}$ & $\begin{array}{l}\text { Higher plasma levels of lysophosphatidylcholine 18:0 are related to a } \\
\text { lower risk of common cancers in a prospective metabolomics study. }\end{array}$ & 2016 \\
\hline 15. & $\begin{array}{l}\text { Ghini V, Unger FT, Tenori L, Turano P, Juhl } \\
\text { H, David KA. }\end{array}$ & $\begin{array}{l}\text { Metabolomics profiling of pre-and post-anesthesia plasma samples of } \\
\text { colorectal patients obtained via Ficoll separation. }\end{array}$ & 2015 \\
\hline 16. & $\begin{array}{l}\text { Zhu J, Djukovic D, Deng L, Gu H, Himmati F, } \\
\text { Abu Zaid M, et al. }\end{array}$ & $\begin{array}{l}\text { Targeted serum metabolite profiling and sequential metabolite ratio } \\
\text { analysis for colorectal cancer progression monitoring. }\end{array}$ & 2015 \\
\hline 17. & Chen L, Zhang C, Gui Q, Chen Y, Yang Y. & \begin{tabular}{|l|} 
Ultra-performance liquid chromatography coupled with quadrupole time- \\
of-flight mass spectrometry-based metabolic profiling of human serum \\
prior to and following radical resection of colorectal carcinoma.
\end{tabular} & 2015 \\
\hline 18. & $\begin{array}{l}\text { Troche JR, Mayne ST, Freedman ND, Shebl } \\
\text { FM, Guertin KA, Cross AJ, et al. }\end{array}$ & $\begin{array}{l}\text { Alcohol consumption-related metabolites in relation to colorectal cancer } \\
\text { and adenoma: Two case-control studies using serum biomarkers. }\end{array}$ & 2016 \\
\hline 19. & $\begin{array}{l}\text { Farshidfar F, Kopciuk KA, Hilsden R, } \\
\text { Mcgregor SE, Mazurak VC, Buie WD, et al. }\end{array}$ & A quantitative multimodal metabolomic assay for colorectal cancer. & 2018 \\
\hline 20. & Zhang F, Zhang Y, Zhao W, Deng K, Wang Z. & $\begin{array}{l}\text { Metabolomics for biomarker discovery in the diagnosis, prognosis, } \\
\text { survival and recurrence of colorectal cancer : a systematic review. }\end{array}$ & 2017 \\
\hline 21. & $\begin{array}{l}\text { Long Y, Sanchez-Espiridion B, Lin M, White } \\
\text { L, Mishra L, Raju GS, et al. }\end{array}$ & $\begin{array}{l}\text { Global and targeted serum metabolic profiling of colorectal cancer } \\
\text { progression. }\end{array}$ & 2017 \\
\hline 22. & $\begin{array}{l}\text { Brown DG, Rao S, Weir TL, O’Malia J, Bazan } \\
\text { M, Brown RJ, et al. }\end{array}$ & $\begin{array}{l}\text { Metabolomics and metabolic pathway networks from human colorectal } \\
\text { cancers, adjacent mucosa, and stool. }\end{array}$ & 2016 \\
\hline 23. & $\begin{array}{l}\text { Ogino S, Nishihara R, Vanderweele TJ, Wang } \\
\text { M, Nishi A, Lochhead P, et al. }\end{array}$ & $\begin{array}{l}\text { The Role of Molecular Pathological Epidemiology in the Study of } \\
\text { Neoplastic and Non-Neoplastic Diseases in the Era of Precision Medicine }\end{array}$ & 2016 \\
\hline 24. & $\begin{array}{l}\text { Djukovic D, Zhang J, Raftery D. Colorectal } \\
\text { cancer detection using targeted LC-MS } \\
\text { metabolic profiling. }\end{array}$ & Colorectal cancer detection using targeted LC-MS metabolic profiling. & 2018 \\
\hline 25. & $\begin{array}{l}\text { Perttula K, Schiffman C, Edmands WMB, } \\
\text { Petrick L, Grigoryan H, Cai X, et al. }\end{array}$ & $\begin{array}{l}\text { Untargeted lipidomic features associated with colorectal cancer in a } \\
\text { prospective cohort }\end{array}$ & 2018 \\
\hline
\end{tabular}




\section{Results}

\section{Clinical applications in CRC}

The main practical application would be searching for biomarkers in order to obtain an early diagnosis and proper postoperative follow-up. A well-known multicenter study developed in China and the United States find a common 15 metabolite pattern that could allow assessment of the recurrence rate and survival of the patients after surgical intervention or chemotherapy. In this study Qui et al. analyse by gas chromatography 376 surgical specimens from three chinese centers and City of Hope Comprehensive Cancer Center in U.S. [10].

A systematic review published in march 2017 selected from a total of 156 papers referring the human CRC metabolomics a final list of 23 eligible articles. From these 16 had as the main subject of study diagnosis, 2 were about prognosis and survival, 4 were focused on diagnosis, prognosis and survival and 1 on diagnosis, prognosis, survival and recurrence. The studied biologic material was serum samples (11 studies), urine in 4 studies, tissue samples in 9 studies, exhaled air - 1 study, feces -1 study, 2 studies combined serum and tissue samples and 1 study combined feces and tissue samples. The results showed a good discrimination of the CRC patients compared with healthy subjects, even in stage I TNM patients. One study (based on urine metabolites) showed an acceptable staging in "early", "intermediate" and "advanced" (metastatic) stage, with a poor discrimination between intermediate studies. In contrast, 2 tissue samples-based studies showed a pretty exact TNM staging (T1/2, T3 and T4). Regarding the prognosis and recurrence implications the paper cites the above mentioned of Qiu et al. (the 15-metabolite pattern) as well as the study of Cheng et al. (see below) which identifies biomarkers with prognosis and survival implications (kynurenate, 2-aminobutyrate, succinate, p-cresol, putrescine and fumarate in early diagnosis and stages) [11]. The study of Tan et al. failed to stratify TNM stages but showed that $\beta$-hydroxibutyrate increased continuously from stage I to stage IV while tryptophan and indoleacrylic acid were continuously decreasing through stage I to stage IV cases [12]. In his 2016 study, Farshidfar identifies a 40 metabolites model that change with increasing T-stage and a 17 compound metabolomic model that could categorize the N-Stages [13].

In the study of Zhu, Djukovic et al. from 2015, targeted liquid chromatography was used to monitor disease progression of $20 \mathrm{CRC}$ patients. It demonstrates the superiority of a 5-metabolite fingerprint (succinate, N2,N2-dimetihylguanosine, adenine, citraconic acid and 1-methylguanosine) in monitoring the evolution of CRC patients when compared with current markers (particularly the carcinoembryonic antigen - CEA). Sensitivity of the proposed model was 0.83 , specificity of 0,94 , area under ROC curve AUROC of 0.91 vs. 0.75, 0.76 and 0.80 respectively for the CEA parameters. [14].

The study of Chen et al. [15] collected serum samples from 20 CRC patient prior to surgery and one month after the radical intervention and compared them with samples from 20 healthy volunteers. The study identified potential biomarkers from three classes: phosphatidylcholines (PCs), lysophosphatidylcholines (LPCs) and diacylglycerols (DAGs) and tried to establish if these metabolites are modified regarding the pre-operative and post-operative status of the patient. It seems no statistical difference was found in these biomarkers.

\section{Metabolic pathways}

The logical explanation for the changes in the levels of metabolites in some biologic fluid (either increased or decreased levels) is the alteration of the malignant cell metabolism, high rate of proliferation and destruction of cell membrane. According to the type of metabolism affected we can summarize the changes in metabolic pathways found in the literature.

1. Carbohydrate metabolism. The metabolism products are the result of the processes of glycolysis, the tricarboxylic acid cycle and anaerobic respiration. Arabitol, galactose, mannose and pyruvate were constantly found with low serum levels. Lactate, a product of anaerobic glycolysis, was found increased both in tissue studies and serum studies. Galactose, glucose and galactitol had a decreasing trend in all studies [11]. Pyruvate, an important intermediate in glycolisis had higher values than healthy controls, while other intermediates of the TCA cycle like fumarate and cis-aconitate had lower values in the chineseamerican study of Tan et al. [12]. By contrast, an MD Anderson Cancer Center group study found that a higher level of D- mannose was associated with increased risks of adenoma polyps and CRC [16].

2. Lipid metabolism. Increased levels of several lipid metabolites were found: lactate, 2-aminobutyrate, choline, hydroxybutyrate, succinate, acetate, oleic acid, glycochenodeoxycholate and phosphocoline. There was some contradictions regarding for example the arachidonic acid (high levels in one study and low levels in another) [11]. We can also include in this subchapter the cell membrane lipids changes. Most phosphatidylcholines (PCs) were significantly elevated, most of the lysophosphatidylcholine (LDC) and diacylglycerol (DAG) serum levels increased. We should mention that saturated fatty acid LPC were elevated while those containing polyunsaturated fatty acids were lowered in CRC patients (explanation below). [15] Tan on the other hand finds that four lysophosphatidylcholines were decreased. This observation was reported to be associated with body weight loss and activated inflammatory status in cancer patients [12]. Pertulla et al. in a Berkley University paper suggest that some ultra-long chain fatty acids (ULCFAs) and related fatty acids, appear to be products of disease progression and, therefore, could be useful diagnostic biomarkers for early detection of CRC [17].

3. Aminoacid metabolism. According to the review mentioned above, it concludes that 7 amino acids have consistent results in the evaluated trials: histidine, methionine and tryptophan were decreased while glutamic acid, proline / L-proline, iso-glutamine and putrescine were increased. Other metabolites have contradictory results. 
Glycine was increased in tissue in two studies and low in serum in two other studies. Taurine was elevated in 3 studies and decreased in another study [11]. Geijsen et al. also find the plasma level of taurine increased and the levels of valine and leucine decreased. Decreased blood levels of branched-chain aminoacids (BCAAs) could reflect increased requirement for amino acids due to the high protein turnover in the malignant setting [18].

4. Nucleotides. Uracil was increased in tissues in 3 studies, feces (1 study), low in urine (1 study) [11]. Adenyl succinate (purine metabolism) was found to be increased in tumor tissue of CRC patients versus normal mucosa [19].

5. Other significant metabolites. Carnitine and hypoxanthine had high values in several studies in CRC patients and phenol and urea low values. Other metabolites (eg P-crezole - contradictory values in urine in two studies). [11] In the MD Anderson study mentioned above by Long et al, lower levels of xanthine and hypoxanthine were associated with higher risks of adenomas and CRC [16]. In a 2019 multicenter study (Geijsen et al.) on 268 patients with CRC and 353 controls, the level of hypoxanthine was reduced. The authors explanation was that hypoxanthine (as taurine) is an antioxidant and increased levels reported in their study may be the result of increased oxidative stress, which is recognized as an important process in carcinogenesis. They suggest that inconsistent hypoxanthine levels may be caused by red blood cell hemolysis during the preparation of serum samples utilized in the Long study in contrast to plasma used in the present analysis [18].

Knowledge of complex metabolic pathways may explain certain changes in the metabolomic profile by searching for specific cellular processes. For example, decreasing LPC values containing polyunsaturated fatty acids, in contrast to the opposite trend of PCs containing polyunsaturated fatty acids, demonstrated in the study of Chen et al. LPCs are produced by PC degradation. Phospholipase A2, which plays an important role in the catalyzing of PC hydrolysis in LPC, has an antitumor role in various cancers and its low activity in CRC patients can contribute to lowering LPCs [15].

The practical application of knowledge of these pathways will lead to the development of metabolite models that play a role in early diagnosis. The study of Farshidfar et al. (University of Calgary) published in 2018 distinguishes a model of 48 metabolites with an AUROC of 0.98 , a sensitivity of $93 \%$ and a specificity of $95 \%$. It analysed samples from a prospective cohort of 63 CRC patients, 31 patients with adenoma and 81 diseasefree controls. The same model manages to distinguish between adenomatous polyps and healthy subjects [20]. The study of Deng et al. published in 2016 validated a urine-based metabolomic diagnostic test for the detection of adenomatous polyps, PolypDx ${ }^{\mathrm{TM}}$, that was originally developed and validated using 1000 samples from Canadian Cohort, on Chinese population of 1000 participants. This model measures the concentrations of three metabolites in urine samples. The AUC of 0.717 , sensitivity of $82.6 \%$ and specificity of $42.4 \%$ were better than the fecal-based tests [21]. Jing et al. use a 21 metabolite model based on a dried blood spot that showed significant difference between 81 polyp and $85 \mathrm{CRC}$ patients (sensitivity of $81.18 \%$ and specificity of $83.95 \%$ ) [22].

The most impressive results seem to come from the japanese study of Nishiumi et al. The study included 282 stage 0/I/II CRC cases and 291 healthy participants. The AUC, sensitivity, and specificity values of this metabolomic model for detecting stage 0/I/ II colorectal cancer were $0.996,99.3 \%$, and $93.8 \%$, respectively. The model's sensitivity and specificity values for each disease stage were $>90 \%$, and surprisingly, its sensitivity for stage 0 , specificity for stage 0 , and sensitivity for stage II disease were $100 \%$ [23].

Another japanese study (Hata et al) focuses on a single metabolite as a potential biomarker for early detection of CRC. It is a member of the family of longchain (28-carbon) hydroxy fatty acid metabolites referred to as hydroxylated polyunsaturated ultra-long-chain fatty acids (hPULC-FAs) or gastrointestinal tract acids (GTAs) GTA 446 (molecular weight $446 \mathrm{kDa}$ ) - C28H46O4. By analysing serum samples from $225 \mathrm{CRC}$ patients and 916 healthy volunteers, the authors concluded that GTA-446 does not reflect the presence of a tumour, but susceptibility to $\mathrm{CRC}$, and leads to identifying high-risk CRC patients with high sensitivity. This statement has high clinical importance for encouraging colonoscopy [24].

Another interesting approach proposed by other scientists is to associate clinical factors, such as gender, age, medication, and substance status as factors that could strengthen the metabolite biomarker models. Djukovic et al. in a 234 subject study (66 CRC, 76 polyp patients and 92 healthy controls) finds a 13 metabolite model for differentiating between CRC patients and healthy control and 14 metabolite biomarkers selected for the separation of CRC cases from polyps. Four general clinical factors (age, gender, smoking, and alcohol status) were added to the model and the result was an excellent AUROCs (0.93-0.95, respectively) for differentiating CRC patients from healthy controls or polyp patients [25].

\section{Predisposing factors and early diagnosis}

Another direction of research is the study of predisposing factors of cancer from the metabolomic perspective. Epidemiological studies suggest the role of obesity, dyslipidemia, hyperinsulinemia, disruption of glucose homeostasis and elevated Insulin Growth Factor (IGF) as risk and prognostic factors in cancer. A Polish study review investigated from the metabolomic point of view three main axes (dyslipidemia, adipokines and IGF). None of the three lines was established as an individual risk factor, while the association of the three was shown to increase the risk of cancer and the mortality rate for both sexes [26].

One American-British study attempted to demonstrate the possible relationship between a known carcinogen alcohol and CRC. Previous metabolism-related metabolites have been investigated and their possible association with adenoma and CRC has been investigated. The study was 
based on two populations: one of 252 incidental CRC cases and 250 controls from over 150000 participants enroled between 1993 and 2001 in the Prostate, Lung, Colorectal and Ovarian Cancer screening Trial, the other of 244 CRC cases and 231 controls form the Navy Colon Adenoma Study of Maryland. The conclusion of the study is that this association does not exist [27].

The MD Anderson group found that low levels of xanthine and hypoxanthine in smoking patients had a joint effect with smoking in adenoma polyps and CRC risk. Obese patients with the same metabolic changes had an elevated adenoma polyps risk. High levels of D-mannose had joint effects with smoking on CRC risk. Notably, there was a significant interaction between the BMI and the D-mannose level in modifying CRC risk [16].

A German prospective study, with average follow-up of 8.3 years, investigated the association between the prediagnosis levels of 120 metabolites and the risk of breast, prostate and colorectal cancer. The study population was a subset of 163 colorectal cancer patients from the over 25000 population of EPIC-Heidelberg study. The findings of the study are that changes in plasma lipid composition precede the appearance of neoplasia for several years and that tumor changes appear to cause a global change in lysophosphatidylcholine metabolism (consistent with the ones outlined above). It seems that the high level of LPC 18: 0 (unsaturated fatty acids) would be a protective factor $[15,28]$. This could be a possible screening strategy in the future.

\section{Discussions}

\section{Integrative approach}

Another trend in this area of research is to establish the link between genomics and metabolomics, between genome and phenotype. The latest trends of classifying CRC subtypes are based on genetic and epigenetic changes in relation to the molecular pathways that lead to its pathogenesis. In this sense, there is a subtype due to chromosomal instability, microsatellite instability, posttranslational methylation changes. More recently, following the ATLAS Project of the Human Genome, CRC is divided according to the frequency of mutations in hypermutated or non-hypermutated forms. These have different clinical implications regarding prognosis (eg chromosomal instability forms are associated with a worse prognosis regardless of the stage or type of treatment), others may explain the lack of response to some treatments (eg K-Ras and N-Ras) [3, 10, 29]. KRAS mutations occur in $35 \%$ to $45 \%$ of patients with metastatic CRC (mCRC) and predict poor response to EGFR-targeted therapy with cetuximab and panitumumab. An italian-danish paper concludes that mCRC before third-line therapy with cetuximab and irinotecan shows a signature of altered energy metabolism, which may reflect an increased gluconeogenesis and an accumulation of 3-hydroxybutyrate [30]. There are other studies that combine transcriptomics and metabolomics regarding CRC cell lines which express either wild type (wt) KRAS or the oncogenic KRAS (mt). Consistent with the transcriptional data, protein synthesis and cell proliferation were significantly higher in the mtKRAS cells. Targeted metabolomics analysis also confirmed the metabolic reprogramming in mtKRAS cells. Interestingly, mtKRAS cells were highly transcriptionally responsive to EGFR activation by TGF $\alpha$ [31].

Regarding these subjects molecular pathologic epidemiology (MPE) has emerged as an integration of molecular pathology and epidemiology. MPE studies have shown that smoking is a risk factor for a colorectal cancer subtype described as microsatellite instability (MSI) or CpG island methylator phenotype-high while obesity is a risk factor for non-MSI-high subtype of colorectal cancer. Thus, under the MPE paradigm, smoking and obesity are risk factors for different disease subtypes. We can drag the conclusion that prevention and early detection are likely different between non-obese heavy smokers and obese never smokers. MPE approach has shown that colonoscopy may not be as effective in smokers as in non-smokers. So in the future we might need tailored preventive strategies $[32,33]$.

One example regarding the complex relation between genotype, proteomics and cancer cell metabolism is provided by a canadian group from Quebec. They study the complex interaction between isoforms of uridine diphosphoglucuronosyltransferase 1 (UGT1A_i2s) (produced by alternative splicing of the gene locus), proteomic data of the interaction between UGT1A_i2s and the enzyme of glycolysis pyruvate kinase (PKM) and the cancer cell metabolism. Depletion of UGT1A_i2s proteins in HT 115 cancer cells produce higher glycolitic rate at the expense of mitochondrial respiration and lactate accumulation. Untargeted metabolomics further revealed a significantly altered cellular content of 58 metabolites including many intermediates derived from the glycolysis and TCA cycle pathways, changes that were associated with a greater migration potential [34].

A German-American study analysed in a 2015 paper the metabolic pathway differences between visceral and subcutaneous adipose tissue (VAT and SAT) in CRC patients. They analysed through metabolomic and transcriptomic methods paired samples of serum, VAT and SAT. Compared with SAT, VAT displayed elevated markers of inflammatory lipid metabolism, free arachidonic acid, phospholipases (PLA2G10), and prostaglandin synthesisrelated enzymes. Plasmalogen concentrations were lower in VAT than in SAT, which was supported by lower gene expression of FAR1 (fatty acid reductase), the rate-limiting enzyme for ether-lipid synthesis in VAT [35].

Metabolomics (study of small intermediate molecules) of the disease presents a momentary picture, a snapshot of metabolic disturbances accompanying colorectal cancer. Several studies have demonstrated differences between normal and tumor tissues. Generally, metabolites associated with the tricarboxylic acid cycle appear to be found to have a lower level in malignant tissues, while urea, purine, pyrimidine, amino acid and choline intermediates were increased, more likely related to the elevated needs of the malignant cells with rapid division. Liquid stool samples have also been investigated with evidence of changes in fecal metabolites, but it is difficult to tell 
whether these changes are consequential to cancer, is due to microbial populations, or is a predisposing metabolic profile of the patient to develop neoplasia $[3,10,29]$.

\section{Question marks}

The metabolomic studies require special techniques and complex statistic analysis. Due to the complexity of the metabolic processes involved in the tumoral cell and the complex interactions with the normal processes of the organism there are some question marks regarding the stability of the metabolome in time, the scientific accuracy of the data obtained regarding the moment of sample collection (mainly blood or tissue samples), the specificity of the metabolomic signatures of colorectal cancer found in the above mentioned studies compared to other metabolic diseases.

The stability of the metabolome in time. Carayol et al in his paper published in 2015 tried to answer the question about lack of temporal stability of metabolite levels. They collected serum samples two years apart in 27 fasting men from Turin, Italy, and 39 non-fasting women from Utrecht, The Netherlands, all participating in the European Prospective Investigation into Cancer and Nutrition (EPIC) study. The study showed good reproducibility for most of the Biocrates metabolites over a 2-year period, including sphingomyelins (SMs), phosphatidylcholines (PCs), biogenic amines, hexoses and most of the amino acids and acylcarnitines in fasting samples from healthy men from the EPIC cohort. More variability over time was observed in the 140 metabolites when measured in non-fasting samples. The results indicate that a single measurement could be sufficient for hexoses and most of the amino acids, biogenic amines, SMs, and PCs in non-fasting samples, but not for most of the acylcarnitines. Fasting samples are preferable to non-fasting [36]. Related to this subject we searched the literature about the stability of the metabolites in different storage conditions. According to these findings, plasma samples suited for metabolomics analyses should be stored at $\leq-80^{\circ} \mathrm{C}$ for long-term storage due to instability of many of the metabolites in plasma. In fact, the European Prospective Investigation into Cancer and Nutrition (EPIC) consortium in 2005 recommended the storage of plasma in liquid nitrogen for optimal preservation of metabolites, including rapidly degrading metabolites such as vitamin C. Gonzales et al using an elastic net regularized regression model show only minimal deterioration of the plasma metabolome at $-20^{\circ} \mathrm{C}$ up to 1 month. However, majority of the changes appeared at around 12-15 days of storage. This allows scientists to better plan logistics and storage strategies for samples obtained from low-resource settings, where $-80^{\circ} \mathrm{C}$ storage is not guaranteed [37].

Regarding the second issue (the moment of blood sample collection) we found an interesting study of Ghini, Unger, Tenori et al focused on the effects of anesthesia on the metabolic profiles of CRC patients. The study collected blood samples form 70 CRC patients (40 non metastatic and 30 cases with liver metastasis) prior and after drug administration, when the patient was fully anesthetised but before surgery. The two groups of samples were well discriminated, the signals of some molecules are visible in the spectra (Etomidate, Propofol, Cefuroxime). A general decrease in metabolites concentration was observed and it can be reasonably attributed to a slower metabolism under anesthesia. The increased levels of a few molecules are more difficult to interpret but they might be directly related to the effect of some of the medical treatments. The authors logical conclusion is that post-anesthesia samples are not very suitable for standard metabolomic studies [38].

Last, we focused on the complex relationship between CRC and other metabolic diseases, mainly obesity and diabetes, from the metabolomic point of view. Most articles found searched the relationship between obesity, dyslipidemia and CRC. In the aetiopathogenesis of CRC in over-weight/obese patients, fat tissue is said to be of high importance for the processes of neoplastic transformation. Fat tissue is treated like glandular tissue, performing endocrine, paracrine and autocrine functions, regulating triglyceride metabolism, influencing the coagulation system and inhibiting the anti-lipolytic effect of insulin [26]. Findings on the relationship between the levels of total cholesterol and triglycerides and the risk of CRC have been inconsistent. For example in a large-scale cohort study performed by Ulmer et al high serum TG concentrations were found to be correlated with a high risk of rectal cancer $(\mathrm{HR}=1.56,95 \% \mathrm{CI}: 1.00-2.44)$ in men and women combined (as well as for lung, gynaecological cancers and thyroid cancer) but no other correlation was found (in particular colon cancer) [39]. In the metabolic syndrome and cancer (Me-Can) project 2834 men and 1861 women were diagnosed with colorectal cancer. In men, a significant association was observed between the TG level and CRC (RR $=1.17,95 \% \mathrm{CI}: 1.06-1.28)$ and a modest positive association was observed for total cholesterol and CRC. However, no relationship between these factors was found in women [40]. Most of the studies in this area have indicated that leptin may potentiate the growth of cancer cells in vitro, while adiponectin has an opposite effect [26]. As for cancers, recent studies try to define a metabolomic signature for obesity. Elevated levels of branched-chain aminoacids (BCAAs) (leucine, isoleucine, and valine) and aromatic AAs (phenylalanine, tyrosine, tryptophan and methionine), as well as some of their tissue metabolites, have been detected in both subjects with obesity and diabetes, whereas glutamine and glycine levels are decreased. Nucleotides, nucleosides, and their metabolites, namely, uridine and uric acid, vary considerably, depending on the degree of insuline resistance in obese subjects. The concentrations of glucose, fructose, mannose, xylose, gluconic acid, glucuronic acid, glycerol and lactate in plasma are usually increased, whereas the concentrations of glycerol-3-phosphate and other metabolites are decreased in obese men. Serum concentrations of lysoPCs show a robust inverse correlation with BMI, body weight and waist and hip circumference [41].

There is a significantly elevated risk for proximal colorectal cancer in men suffering from type 2 diabetes mellitus and no significant increase in risk in women with this condition [42]. Other studies have indicated that 
diabetes mellitus type 2 is a risk factor for distal CRC [43] and both proximal and distal CRC [44]. However, several studies have revealed a decrease in cancer risk in diabetic patients treated with metformin, an anti-diabetic agent [41, $45,46]$. Higher circulating levels of the BCAAs (isoleucine, leucine and valine) are strongly associated with higher type 2 diabetes risk. A large scale genetic and metabolomic study is consistent with a causal role of BCAA metabolism in the aetiology of type 2 diabetes [47]. A combination of individual fatty acids, characterised by high concentrations of linoleic acid, odd- chain fatty acids, and very long-chain fatty acids, was associated with lower incidence of type 2 diabetes. The specific fatty acid pattern may be influenced by metabolic, genetic, and dietary factors [48].

\section{Conclusions}

In conclusion - the metabolomics can provide a useful and non-invasive tool for early diagnosis, probably staging and prognosis in colorectal cancer, already existing studies showing metabolomic models with statistical power much better than current existing markers. There is also hope of using them as a screening method.

\section{References}

1. Facts CC. Colorectal Cancer. 2016.

2. SIEGEL RL, MILLER KD, FEDEWA SA, AHNEN DJ, MEESTER RGS, BARZI A et al. Colorectal cancer statistics, 2017. CA Cancer J Clin [Internet]. 2017 May 6 [cited 2017 Jun 18]; 67(3):177-93. Available from: http://doi.wiley. com/10.3322/caac.21395

3. FARSHIDFAR F, WELJIE AM, KOPCIUK K, BUIE WD, MACLEAN A, DIXON E, et al. Serum metabolomic profile as a means to distinguish stage of colorectal cancer. 2012.

4. Metabolomics $\mid$ definition of metabolomics by Medical dictionary [Internet]. [cited 2018 Sep 23]. Available from: https://medical-dictionary.thefreedictionary.com/ metabolomics

5. DAVISON KK, BIRCH LL. NIH Public Access. 2008; 64(12):2391-404.

6. Metabonomics | definition of metabonomics by Medical dictionary [Internet]. [cited 2018 Sep 23]. Available from: https://medical-dictionary.thefree dictionary.com/metabonomics

7. Metabolome / definition of metabolome by Medical dictionary [Internet]. [cited 2018 Sep 23]. Available from: https://medical-dictionary.thefreedictionary.com/ metabolome

8. Manuscript A. NIH Public Access. 2015; 1-9.

9. BEGGS AD, DILWORTH MP. Surgery in the era of the ' omics revolution. 2015.

10. QIU Y, CAI G, ZHOU B, LI D, ZHAO A, XIE G, et al. A Distinct Metabolic Signature of Human Colorectal Cancer with Prognostic Potential. 2014; 20(8):2136-47.

11. ZHANG F, ZHANG Y, ZHAO W, DENG $\mathrm{K}$, WANG Z. Metabolomics for biomarker discovery in the diagnosis, prognosis, survival and recurrence of colorectal cancer: a systematic review. 2017; 8(21): 35460-72.

12. TAN B, QIU Y, ZOU X, CHEN T, XIE G, CHENG Y, et al. Metabonomics Identi fi es Serum Metabolite Markers of Colorectal Cancer. 2013.

13. FARSHIDFAR F, WELJIE AM, KOPCIUK KA, HILSDEN R, MCGREGOR SE, BUIE WD, et al. A validated metabolomic signature for colorectal cancer: exploration of the clinical value of metabolomics. 2016; (August):1-10. Available from: http://dx.doi.org/10.1038/bjc.2016.243

14. ZHU J, DJUKOVIC D, DENG L, GU H, HIMMATI F, ABU ZAID $\mathrm{M}$, et al. Targeted serum metabolite profiling and sequential metabolite ratio analysis for colorectal cancer progression monitoring. Anal Bioanal Chem. 2015; 407(26):7857-63.

15. CHEN L, ZHANG C, GUI Q, CHEN Y, YANG Y. Ultra-performance liquid chromatography coupled with quadrupole time-of-flight mass spectrometrybased metabolic profiling of human serum prior to and following radical resection of colorectal carcinoma. Mol Med Rep. 2015;12(5):6879-86.

16. LONG Y, SANCHEZ-ESPIRIDION B, LIN M, WHITE L, MISHRA L, RAJU GS, et al. Global and targeted serum metabolic profiling of colorectal cancer progression. Cancer. 2017; 123(20):4066-74.

17. PERTTULA K, SCHIFFMAN C, EDMANDS WMB, PETRICK L, GRIGORYAN $\mathrm{H}$, CAI $\mathrm{X}$, et al. Untargeted lipidomic features associated with colorectal cancer in a prospective cohort 11 Medical and Health Sciences 1112 Oncology and Carcinogenesis. BMC Cancer. 2018 Oct 19; 18(1).

18. GEIJSEN AJMR, BREZINA S, KESKI-RAHKONEN $\mathrm{P}$, BAIERL A, BACHLEITNER-HOFMANN T, BERGMANN MM, et al. Plasma metabolites associated with colorectal cancer: A discovery-replication strategy. Int J Cancer. 2019 Sep 1; 145(5):1221-31.

19. BROWN DG, RAO S, WEIR TL, O'MALIA J, BAZAN M, BROWN RJ, et al. Metabolomics and metabolic pathway networks from human colorectal cancers, adjacent mucosa, and stool. Cancer Metab [Internet]. 2016 [cited 2018 Sep 23]; 4:11. Available from: http://www.ncbi.nlm.nih.gov/pubmed/27275383

20. FARSHIDFAR F, KOPCIUK KA, HILSDEN R, MCGREGOR SE, MAZURAK VC, BUIE WD, et al. A quantitative multimodal metabolomic assay for colorectal cancer. 2018; 1-12.

21. DENG L, FANG H, TSO VK, SUN Y, FOSHAUG RR, KRAHN SC, et al. Clinical validation of a novel urine-based metabolomic test for the detection of colonic polyps on Chinese population. Int J Colorectal Dis [Internet]. 2017;32(5):741-3. Available from: http://dx.doi.org/10.1007/s00384-016-2729-9

22. JING Y, WU X, GAO P, FANG Z, WU J, WANG Q, et al. Rapid differentiating colorectal cancer and colorectal polyp using dried blood spot mass spectrometry metabolomic approach. IUBMB Life. 2017; 69(5): 347-54. 
23. NISHIUMI S, KOBAYASHI T, KAWANA S, UNNO $\mathrm{Y}$, SAKAI T, OKAMOTO $\mathrm{K}$, et al. Investigations in the possibility of early detection of colorectal cancer by gas chromatography/triple-quadrupole mass spectrometry. Oncotarget; Adv Online Publ Page 5 [Internet]. 2017; (Ci):0-4. Available from: http://www.impactjournals. com/oncotarget/index.php?journal=oncotarget\&amp\% 0 Apage $=$ article \&amp $\% 0$ Aop $=$ view $\& a m p \% 0$ Apath $\% 5$ $\mathrm{B} \% 5 \mathrm{D}=15081$

24. HATA T, TAKEMASA I, TAKAHASHI H, HARAGUCHI N, NISHIMURA J. Downregulation of serum metabolite GTA-446 as a novel potential marker for early detection of colorectal cancer. 2017; (May): 1-6. Available from: http://dx.doi.org/10.1038/bjc. 2017.163

25. DJUKOVIC D, ZHANG J, RAFTERY D. Colorectal cancer detection using targeted LC-MS metabolic profiling. In: Methods in Molecular Biology. Humana Press Inc.; 2018. p. 229-40.

26. MUC-WIERZGOŃ M, NOWAKOWSKA-ZAJDEL E, DZIĘGIELEWSKA-GESIAK S, KOKOT $\mathrm{T}$. Specific metabolic biomarkers as risk and prognostic factors in colorectal cancer. 2014; 20(29):9759-74.

27. TROCHE JR, MAYNE ST, FREEDMAN ND, SHEBL FM, GUERTIN KA, CROSS AJ, et al. Alcohol consumption-related metabolites in relation to colorectal cancer and adenoma: Two case-control studies using serum biomarkers. PLoS One. 2016; 11(3):1-14.

28. KÜHN T, FLOEGEL A, SOOKTHAI D, JOHNSON T, ROLLE-KAMPCZYK U, OTTO W, et al. Higher plasma levels of lysophosphatidylcholine 18:0 are related to a lower risk of common cancers in a prospective metabolomics study. BMC Med [Internet]. 2016; 14(1):13. Available from: http://www.biomedcentral. com/1741-7015/14/13

29. BATHE OF, FARSHIDFAR F. From Genotype to Functional Phenotype: Unraveling the Metabolomic Features of Colorectal Cancer. 2014; 536-60.

30. BERTINI I, CACCIATORE S, JENSEN BV, SCHOU J V, JOHANSEN JS, KRUHØFFER M, et al. Metabolomic NMR fingerprinting to identify and predict survival of patients with metastatic colorectal cancer. Cancer Res. 2012 Jan 1;72(1):356-64.

31. CHARITOU T, SRIHARI S, LYNN MA, JARBOUI M-A, FASTERIUS E, MOLDOVAN M, et al. Transcriptional and metabolic rewiring of colorectal cancer cells expressing the oncogenic KRAS G13D mutation. Br J Cancer [Internet]. 2019 [cited 2020 Jan 5]; 121: 37-50. Available from: https://doi.org/10.1038/s41416$\underline{019-0477-7}$

32. OGINO S, NISHIHARA R, VANDERWEELE TJ, WANG M, NISHI A, LOCHHEAD P, et al. The Role of Molecular Pathological Epidemiology in the Study of Neoplastic and Non-Neoplastic Diseases in the Era of
Precision Medicine HHS Public Access. Epidemiology. 2016; 27(4):602-11.

33. LOCHHEAD P, CHAN AT, GIOVANNUCCI E, FUCHS CS, WU K, NISHIHARA R, et al. Progress and opportunities in molecular pathological epidemiology of colorectal premalignant lesions. Am J Gastroenterol. 2014; 109(8):1205-14.

34. Audet-delage Y, ROULEAU M, ROULEAU M, ROBERGE J. No Title.

35. LIESENFELD DB, GRAPOV D, FAHRMANN JF, SALOU M, SCHERER D, TOTH R, et al. Metabolomics and transcriptomics identify pathway differences between visceral and subcutaneous adipose tissue in colorectal cancer patients: the ColoCare study. Am J Clin Nutr [Internet]. 2015 Aug 1 [cited 2017 Nov 14]; 102(2):433-43. Available from: http://ajcn.nutrition.org/cgi/doi/10.3945/ajcn.114.103804

36. CARAYOL M, LICAJ I, ACHAINTRE D, SACERDOTE C, VINEIS P, KEY TJ, et al. Reliability of Serum Metabolites over a Two-Year Period: A Targeted Metabolomic Approach in Fasting and NonFasting Samples from EPIC. Villa E, editor. PLoS One [Internet]. 2015 Aug 14 [cited 2017 Nov 14]; 10(8): e0135437. Available from: http://dx.plos.org/10.1371/ journal.pone.0135437

37. GONZALES GB, DE SAEGER S. Elastic net regularized regression for time-series analysis of plasma metabolome stability under sub-optimal freezing condition. Sci Rep. 2018 Dec 1; 8(1).

38. GHINI V, UNGER FT, TENORI L, TURANO P, JUHL H, DAVID KA. Metabolomics profiling of pre-and post-anesthesia plasma samples of colorectal patients obtained via Ficoll separation. Metabolomics. 2015;11(6):1769-78.

39. ULMER H, BORENA W, RAPP K, KLENK J, STRASAK A, DIEM G, et al. Serum triglyceride concentrations and cancer risk in a large cohort study in Austria. Br J Cancer [Internet]. 2009 Oct 6 [cited 2020 Jan 24]; 101(7):1202-6. Available from: http://www. ncbi.nlm.nih.gov/pubmed/19690552

40. STROHMAIER S, EDLINGER M, MANJER J, STOCKS T, BJØRGE T, BORENA W, et al. Total serum cholesterol and cancer incidence in the Metabolic syndrome and Cancer Project (Me-Can). PLoS One [Internet]. 2013 [cited 2020 Jan 24]; 8(1):e54242. Available from: http://www.ncbi.nlm. nih.gov/pubmed/23372693

41. RANGEL-HUERTA OD, PASTOR-VILLAESCUSA B, GIL ANGEL. Are we close to defining a metabolomic signature of human obesity? A systematic review of metabolomics studies. Metabolomics [Internet]. 123AD [cited 2020 Jan 10]; 1:93. Available from: https://doi.org/10.1007/s11306-019-1553-y 
42. LIMBURG PJ, VIERKANT RA, FREDERICKSEN ZS, LEIBSON CL, RIZZA RA, GUPTA AK, et al. Clinically confirmed type 2 diabetes mellitus and colorectal cancer risk: a population-based, retrospective cohort study. Am J Gastroenterol [Internet]. 2006 Aug [cited 2020 Jan 25]; 101(8):1872-9. Available from: http://www.ncbi.nlm.nih.gov/pubmed/16790032

43. OH S-W, KIM Y-H, CHOI YS, CHANG DK, SON HJ, RHEE P-L, et al. The comparison of the risk factors and clinical manifestations of proximal and distal colorectal cancer. Dis Colon Rectum [Internet]. 2008 Jan [cited 2020 Jan 25]; 51(1):56-61. Available from: http://www. ncbi.nlm.nih.gov/pubmed/18030529

44. YANG YX, HENNESSY S, LEWIS JD. Type 2 diabetes mellitus and the risk of colorectal cancer. Clin Gastroenterol Hepatol. 2005 Jun; 3(6):587-94.

45. SUISSA S, AZOULAY L. Metformin and the risk of cancer: Time-related biases in observational studies. Vol. 35, Diabetes Care. 2012. p. 2665-73.
46. EVANS JMM, DONNELLY LA, EMSLIE-SMITH AM, ALESSI DR, MORRIS AD. Metformin and reduced risk of cancer in diabetic patients. $B M J$ [Internet]. 2005 Jun 4 [cited 2020 Jan 25]; 330(7503): 1304-5. Available from: http://www.ncbi.nlm.nih.gov/ pubmed/15849206

47. LOTTA LA, SCOTT RA, SHARP SJ, BURGESS S, LUAN J, TILLIN T, et al. Genetic Predisposition to an Impaired Metabolism of the Branched-Chain Amino Acids and Risk of Type 2 Diabetes: A Mendelian Randomisation Analysis. PLoS Med. 2016 Nov 1; 13(11).

48. IMAMURA F, SHARP SJ, KOULMAN A, SCHULZE MB, KRÖGER J, GRIFFIN JL, et al. A combination of plasma phospholipid fatty acids and its association with incidence of type 2 diabetes: The EPIC-InterAct case-cohort study. PLoS Med. 2017 Oct $1 ; 14(10)$. 